\title{
Vehicle Dynamics Estimation for Camera-based Visibility Distance Estimation
}

\author{
Clément Boussard, Nicolas Hautière and Brigitte d'Andréa-Novel
}

\begin{abstract}
The presence of an area with low visibility conditions is a relevant information for autonomous vehicle as far as environment sensing is important regarding safety. In this aim, we develop a generic sensor of visibility using an onboard camera in a vehicle. Our approach consists in estimating the range to the most distant object belonging to the plane of the road having at least $5 \%$ of contrast. The originality of this approach lies in the fact that the depth map of the vehicle environment is obtained by aligning the road plane in successive images. This algorithm exploits the dynamics of the vehicle which is given or observed from proprioceptive sensors classically available on public vehicles. In this paper, we present the principle of our approach in terms of image processing and explain how the vehicle dynamics takes part in it with a sensitivity study.
\end{abstract}

\section{INTRODUCTION}

To increase safety onboard, for example by warning the driver in case of unadapted behavior regarding the road or weather conditions, the need for driving environment sensing is crucial. In particular, environment perception using cameras, lidars and radars enables to segment the driving area, to detect the obstacles and the vulnerable road users, or to detect the local weather conditions. In this paper, our objective is to locally measure the visibility range using an on-board camera, in the aim of detecting low visibility conditions due to degraded weather conditions.

Different studies about visibility distance measurement exist, among which we can find:

- A method using detection of lane markings: Pomerleau [1] estimates the visibility distance by measuring the contrast attenuation of lane markings at different distances in front of the vehicle.

- A mono-camera method adapted to fog using Koschmieder's model [2]. We obtain, under daytime foggy weather, an estimation of the meteorological visibility distance [3].

- A method using stereo-vision: this method is generic and not limited to fog. Thanks to stereo-vision, a good quality depth map is computed [4]. The distance to the furthest point of the road surface with a contrast greater than $5 \%$ gives the visibility distance [5].

This work has been partly supported by the FP6 REACT project and the ANR DIVAS project

C. Boussard and B. d'Andréa-Novel are with Robotic center, Ecole Nationale Supérieure des Mines de Paris, 75006 Paris, France clementboussardefreesurf.fr and brigitte.dandrea-noveleensmp.fr

N. Hautière is with Laboratoire Central des Ponts et Chaussées, LCPC, 75015 Paris, France nicolas.hautiere@lcpc.fr
Contrary to the method using Koschmieder's model, the stereo-vision method does not make any difference between the geometric and atmospheric visibility distance. Indeed, if there is a curve or a uphills where the visibility is reduced due to physical reasons, the visible road surface will be limited by the road geometry [6]. In these cases, the visibility distance calculated will be the geometric one. In this way, the different methods are complementary.

The method we design is as generic as the one based on stereo-vision but uses only one camera and aims at supplementing the method based on Koschmieder's model. To achieve this goal, we estimate the distance of the furthest object which is part of the road plane with a contrast higher or equal to $5 \%$. This method takes into account the definition of the meteorological visibility distance given by the CIE [7] and is decomposed in three parts:

1) Creation of a pseudo-depth map of the vehicle environment by aligning the road plane in the successive images.

2) Creation of a contrast map.

3) The visibility distance is obtained by taking the furthest point (depth map) with a contrast greater than $5 \%$ (contrast map).

\section{PSEUDO-DEPTH MAP CONSTRUCTION}

Through use of a single camera, it is impossible to get directly the depth in images. But we can calculate with perspective projection the distance of points belonging to the road. The most generic way to determine the road plane is to align two successive images. Objects belonging to the road plane are at the same place from the first image to the second one, at the opposite, verticals objects are deformed. In general, successive images alignment is made with classical image processing techniques, e.g. [8]. These methods consist in matching objects in the two images. In our degraded visibility context, this approach is not well adapted because local contrasts are strongly deteriorated. The originality of our approach is to align two images with the knowledge of the motion of the camera, which is observed or measured with proprioceptive sensors.

\section{A. Successive Images Alignment}

1) Image acquisition: In the coordinate system of the camera frame, the position of a pixel in the image plane is given by its coordinates $(u, v)$. The image optical center is denoted $\left(u_{0}, v_{0}\right)$ in the image frame and considered as the image center. The transformation between the vehicle frame (with origin at the center of gravity of the vehicle) and 


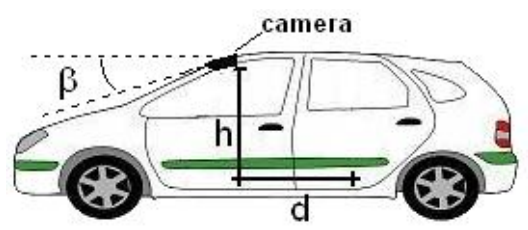

Fig. 1. Position of the camera in the vehicle

the camera frame, is represented by a vectorial translation $\vec{t}=d \vec{X}+h \vec{Z}$ (Fig. 1) and a rotation around the axes $Y$ of angle $\beta$. We denote $T$ the translation matrix and $R$ the rotation matrix. The coordinate change between the image frame and the camera frame can be expressed using a projective matrix $M_{p r o j}$ [9]:

$$
M_{\text {proj }}=\left(\begin{array}{cccc}
u_{0} & 0 & -\alpha & 0 \\
v_{0} & -\alpha & 0 & 0 \\
1 & 0 & 0 & 0
\end{array}\right)
$$

where $\alpha$ represents the ratio between the camera focal length and the size of one pixel. At last, we obtain the transformation matrix $T_{r}$ from the vehicle frame to the image frame:

$$
T_{r}=M_{p r o j} R T
$$

Where $R$ and $T$ are the rotation and translation matrix of the camera position expressed in the vehicle frame.

If $P$ is a point with homogeneous coordinates $(X, Y, Z, 1)$ in the vehicle frame, its homogeneous coordinates in the image frame become:

$$
p=T_{r} P=(x, y, z)^{T}
$$

We can now compute the coordinates $(u, v)$ of the projection of $P$ in the image frame:

$$
\left\{\begin{array}{l}
u=\frac{x}{z}=u_{0}+\alpha \frac{\sin \beta(X+d)-\cos \beta(Z+h)}{\cos \beta(X+d)+\sin \beta(Z+h)} \\
v=\frac{y}{z}=v_{0}-\alpha \frac{Y}{\cos \beta(X+d)+\sin \beta(Z+h)}
\end{array}\right.
$$

\section{2) Creation of a transformed image:}

a) Flat World Assumption: If we consider $I_{1}$ and $I_{2}$ images taken at time $t_{1}$ and $t_{2}\left(t_{2}>t_{1}\right)$, the knowledge of the vehicle motion allows us, thanks to (4), to obtain an estimation of the image $I_{2}$ from the image $I_{1}$. Let $\tilde{I}_{12}$ be this estimated image and $P$ a point whose projection in the image frame belongs to it. Let us assume that this point belongs to the road plane, meaning that if $\left(X_{2}, Y_{2}, Z_{2}\right)$ are the coordinates of this point in the vehicle frame, then $Z_{2}=$ 0 . So the expression of $X_{2}$ and $Y_{2}$ is deduced from (4):

$$
\left\{\begin{array}{l}
X_{2}=\frac{\cos \beta[d U+\alpha h]+\sin \beta[h U-\alpha d]}{\alpha \sin \beta-\cos \beta U} \\
Y_{2}=\frac{-h V}{\alpha \sin \beta-\cos \beta U}
\end{array}\right.
$$

where $U=u-u_{0}$ and $V=v-v_{0}$

b) Vehicle motion: If we know the vehicle motion, we can calculate its movement between time $t_{1}$ and $t_{2}$. As soon as we have points in the vehicle frame (5) we can get new points following this movement (see Fig.2). From the knowledge of the coordinates of a point $P$ and the vehicle

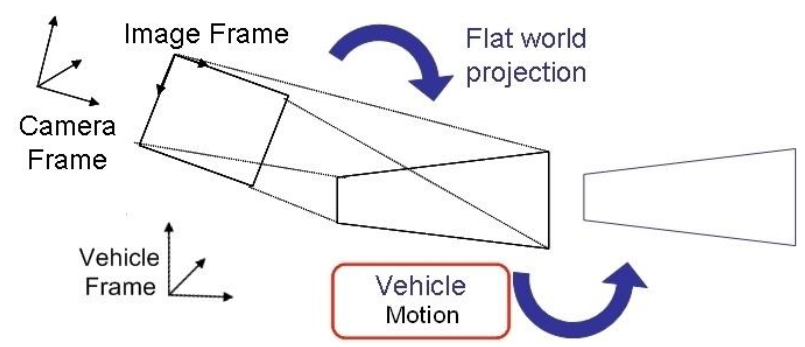

Fig. 2. Motion of the vehicle

dynamics, we can express the coordinates of the point $P$ in the camera frame at time $t_{1}$ :

$$
\left(x_{12}, y_{12}, z_{12}\right)^{T}=T_{r} M\left(X_{2}, Y_{2}, 0\right)^{T}
$$

where $M$ is the vehicle rotation/translation matrix between the two instants. It represents the vehicle movement.

We obtain the coordinates $\left(u_{12}, v_{12}\right)$ of $P$ in the image frame of $I_{1}$ :

$$
u_{12}=\frac{x_{12}}{z_{12}} \quad \text { and } \quad v_{12}=\frac{y_{12}}{z_{12}}
$$

\section{B. Pseudo-disparity Computation between two Images}

We have to match both images. It means that we have to find local correspondences between two neighborhoods from each image. These correspondences are computed via the ZNCC correlation metrics (a comparison of different existing metrics is carried out in [10]). To realize this operation, we have to select a pixel $p_{1}=\left(u_{1}, v_{1}\right)$ in the image $I_{1}$ and another pixel $p_{2}=\left(u_{2}, v_{2}\right)$ in the transformed image $\tilde{I}_{12}$. Then, we define a centered neighborhood $V\left(p_{1}\right)$ around the pixel $p_{1}$ and $V\left(p_{2}\right)$ around the pixel $p_{2}$ in which we are computing the ZNCC correlation score:

$$
Z N C C\left(p_{1}, p_{2}\right)=\frac{\sum_{V\left(p_{1}\right), V\left(p_{2}\right)} \widetilde{I}_{1} \widetilde{I}_{2}}{\sqrt{\sum_{V\left(p_{1}\right), V\left(p_{2}\right)} \widetilde{I}_{1}^{2} \sum_{V\left(p_{1}\right), V\left(p_{2}\right)} \widetilde{I}_{2}^{2}}}
$$

where

$$
\begin{aligned}
& \left.\widetilde{I}_{1}=\left(I_{1}\left(u_{1}+i, v_{1}+j\right)-\bar{I}_{1}\right)\right) \\
& \left.\widetilde{I}_{2}=\left(I_{2}\left(u_{1}+i, v_{1}+j\right)-\bar{I}_{2}\right)\right)
\end{aligned}
$$

with $I_{i}(u, v)$ the grey level value of the pixel $(u, v)$ of the image $I_{i}$ and $\bar{I}_{i}$ the mean value of $I_{i}(u, v)$ in $V\left(p_{i}\right)$.

The more the correlation score is close to 1 , the more we can consider these two neighborhoods as identical. Working on a single pair of $\left(p_{1}\right.$ and $\left.p_{2}\right)$ limits our study. Indeed, some matching errors can occur and a pixel belonging to the road can be incorrectly matched in the image $\tilde{I}_{12}$. That's why we have to extend our study zone. To do it, we have defined a search window. The correlation neighborhood in image $I_{1}$ is centered on a point of interest. The correlation neighborhood in the image $\tilde{I}_{12}$ is centered successively around a pixel varying in a search frame (this search frame is centered on the pixel $p_{1}$ of image $I_{1}$ ). This principle is schematized in Fig. 3.

As soon as the scanning of the search window is done, 


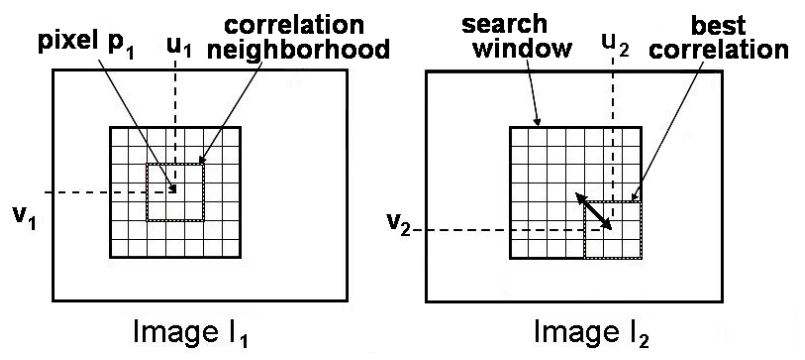

Fig. 3. Correlation neighborhood and search window

we keep the position $\left(u_{2}, v_{2}\right)$ of the pixel with the best correlation score. With these two positions, we calculate a disparity:

$$
d=\sqrt{\left|u_{1}-u_{2}\right|^{2}+\left|v_{1}-v_{2}\right|^{2}}
$$

After that, we only kept points with a small disparity, in considering them as points belonging to the road plane. This process allows us to create a map of pixels belonging to the road plane. Therefore a new map can be created as soon as a new transformed image is calculated. In our application we create a new one when the vehicle movement is big enough to get a good comparison between the two images.

\section{Visibility range estimation}

To estimate the visibility distance, we combine the measurement of contrasts higher than $5 \%$ with the map of the pixels belonging to the road plane. In this aim, we locally process the contrast of image points belonging to the road plane by scanning it from top to bottom starting from the horizon line. As soon as we find a point with a contrast greater or equal to $5 \%$, the process stops and the visibility distance is the distance of this point given by (9).

We can see in Fig. 4 the result of the process. The image on top represents an image taken under foggy weather condition. The bottom right image is the classification result of the successive image alignment and the pseudo-disparity computation. The road elements are in black and the nonroad element in white. We can see that the road sign which is a vertical object is not detected as part of the road. The bottom left image is the $5 \%$ contrast map of the image.

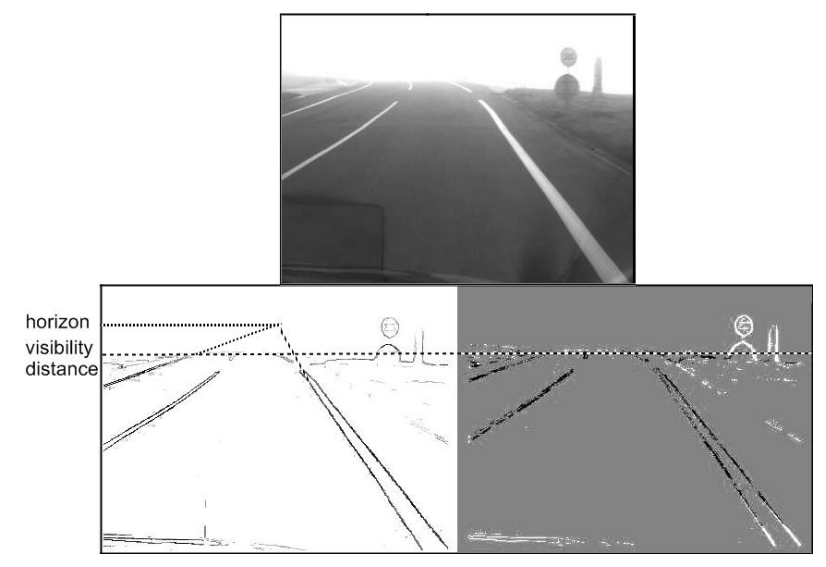

Fig. 4. Image from the camera (up) - $5 \%$ contrast map (left) - Road/Nonroad image (right)
The comparison of this two calculated image allows us to determine the visibility distance.

1) Pseudo-depth map construction: As far as we can say that a pixel of coordinates $(u, v)$ belongs to the road plane, we can express the distance $d$ of this pixel (9) :

$$
d=\left\{\begin{array}{ll}
\frac{\lambda}{v-v_{h}} & \text { if } v>v_{h} \\
\infty & \text { if } v \leq v_{h}
\end{array} \quad \text { where } \lambda=\frac{H \alpha}{\cos ^{2} \beta_{0}}\right.
$$

where $H$ denotes the mounting height of the camera, $\alpha$ the ratio between the camera focal length and the size of one pixel, $\beta_{0}$ the camera pitch angle and $v_{h}$ the position of the horizon in the image $\left(v_{h}=v_{0}-\alpha \tan \beta_{0}\right)$.

2) Contrast estimation: We adapted Köhler's binarization technique [13] in order to measure the local contrasts of images. The idea is to find region separeted by borders and then to locally measure the contrast along the associated border. This method measure the contrast locally in the image. Details of the method can be found in [5].

\section{SENSITIVITY TO VEHICLE DYNAMICS}

\section{A. Problem statement}

In the previous section, we have seen that the vehicle dynamics is a need for our visibility estimation method. Indeed, if we know the six degrees of freedom, the three rotations (roll, pitch, yaw) and the three translations (longitudinal $T_{x}$, lateral $T_{y}$ and vertical $T_{z}$ ), we can do the successive images transformations. Available sensors that allow vehicle dynamics estimation are odometers and an Inertial Measurement Unit (IMU):

- The odometers give the numbers of rotations done by the wheels.

- The IMU gives angular speed of the three rotations axis of the vehicle (roll, pitch, yaw) and accelerations of the three axis of the vehicle $(X, Y, Z)$.

At first sight, the odometers and the IMU should give us the knowledge of the six degrees of freedom that we need. Indeed, if we consider that the wheel radius is constant, we can have an estimation of the distance covered by this wheel, meaning by the vehicle. Moreover, the numerical integration of the angular speed given by the IMU gives an estimation of the relative angular variations between two instants.

Our idea will be to realize on-line estimation of the state variables which are strictly necessary to describe the vehicle dynamics in the different actual driving situations. More precisely we want to know if the knowledge of all the degrees of freedom is really necessary for our successive images alignment. The aim is to eliminate some of the degrees of freedom which are not relevant in our process. To do it, we use the notion of sensitivity [11]. If we just look at the nature of the degrees of freedom, we have angles expressed in radians and distances expressed in meters. So we can not directly compare them. The sensitivity allows us to compare the different contributions of the degrees of freedom using simulated scenarii. 
1) Sensitivity criteria: Let $M_{\text {vehicule }}\left(T_{x}, T_{y}, T_{z}, \theta, \psi, \phi\right)$ be the motion function of the vehicle between time $t_{1}$ and $t_{2}$. From (5) we can compute the coordinates $\left(X_{2}, Y_{2}, Z_{2}\right)$ of the point in the world frame at time $t_{2}$.This brings us to:

$$
\left\{\begin{array}{l}
u_{2}=\frac{x_{2}}{z_{2}}=f_{u}\left(T_{x}, T_{y}, T_{z}, \theta, \psi, \phi, u, v\right) \\
v_{2}=\frac{y_{2}}{z_{2}}=f_{v}\left(T_{x}, T_{y}, T_{z}, \theta, \psi, \phi, u, v\right)
\end{array}\right.
$$

The detail of (10) is explained in section (II-A.2) through (5) and (4). (10) allows us to say that we have an algebraic relation between $\left(u_{2}, v_{2}\right)$ and $(u, v)$. A sensitivity study is done with respect to a criteria (or a cost function). We have to define a criteria that helps us in knowing which degree of freedom influences the most the successive image transformations.

The criteria that seems to be the most important is the pixel displacement. So we define the following criteria:

$$
J(u, v)=\sqrt{\left(u_{2}-u\right)^{2}+\left(v_{2}-v\right)^{2}}
$$

With the help of this criteria we are able to quantify the influence of the different degrees of freedom on the displacement of a pixel $(u, v)$ through the transformation (10).

2) Sensitivity calculation: The parametric sensitivity computation is defined as being the cost function derivative with respect to the studied parameter (12):

$$
S_{p}(u, v)=\frac{\partial J}{\partial p}
$$

Now, we have to compare results obtained in generalizing this kind of computation for all the degrees of freedom. The vehicle dynamics is time varying. The value of the degrees of freedom is not always the same. We have to define some situations in which we compute sensitivity. This process allows us to say that in specific situations (braking, turning, etc.), which degrees of freedom are the most dominating in the image transformations.

\section{B. Preliminary results}

We have designed a prototyping platform [14] with which we can simulate the behavior of a vehicle and its onboard sensors, get exactly their motions and see the results of the successive images transformations. We have defined different scenarii to stimulate all the degrees of freedom and to reproduce some of the classic vehicle behaviors. The initial speed was all the time $30 \mathrm{~km} / \mathrm{h}$ and the different scenarii are the followings:

- Acceleration and braking in a straight line: the acceleration was between $1.5 \mathrm{~m} / \mathrm{s}^{2}$ and $-1.5 \mathrm{~m} / \mathrm{s}^{2}$.

- Right and left oscillation at constant speed: on a two lane road, we move the vehicle from lane to the other.

- Straight line at a constant speed.

- Long right turn: at a constant speed, we turn the wheel to turn along a circle.

Table I shows the maximum value of the normalized sensitivity $\left(\bar{S}_{p}(u, v)=S_{p}(u, v) \times \frac{p}{J}\right)$ we obtain for all the degrees of freedom we consider.

We can see, as expected, that the pixel displacement $J(u, v)(11)$ is less sensitive to the translation $T_{z}$ and the pitch and roll angle, except in the first scenario (acceleration and braking). We can say that, as soon as we are driving at a constant speed, doing a turn or changing lane, the three most important degrees of freedom are the two translations $T_{x}$ and $T_{y}$ and the yaw angle. The others can be neglected.

\begin{tabular}{|c|c|c|c|c|}
\hline & $\begin{array}{c}\text { Acceleration } \\
\text { Braking }\end{array}$ & $\begin{array}{c}\text { Right-Left } \\
\text { oscillation }\end{array}$ & $\begin{array}{c}\text { Constant } \\
\text { speed }\end{array}$ & $\begin{array}{c}\text { Right } \\
\text { turn }\end{array}$ \\
\hline$T_{x}$ & 20 & 15 & 20 & 20 \\
\hline$T_{y}$ & 0 & 8 & 0 & 20 \\
\hline$T_{z}$ & 3 & 2 & 0 & 1 \\
\hline pitch & 10 & 4 & 0 & 4 \\
\hline roll & 0 & 3 & 0 & 1 \\
\hline yaw & 0 & 50 & 0 & 100 \\
\hline
\end{tabular}

TABLE I

NORMALIZED SENSITIVITY FOR THE PIXELS DISPLACEMENTS FUNCTIONS $J(u, v)$ OBTAINED WITH THE DIFFERENT SCENARII

In a real time context, we could compute from (12) numerical approximations of the sensitivities with respect to the different state variables. When some sensitivity values become larger than a predefined thresholds, we decide to estimate the corresponding variables by launching the appropriate estimation algorithm.

\section{VEHICLE DYNAMICS ESTIMATION}

Now that we have realized our sensitivity analysis and we have deduce the most important degrees of freedom to perform correctly our successive image alignment, we will estimate these degrees of freedom based on measurements obtained with an instrumented vehicle.

Vehicle dynamic estimation is done with two kind of sensors: odometers and an inertial measurement unit (IMU).

\section{A. Use of the Vehicle Odometers}

We have seen in the sensitivity analysis that the knowledge of the $x$ and $y$ axis translations are important. Moreover, the acceleration measure has noise. The double integration of these accelerations will accumulate errors due to the noise and then it will create a bias in the estimation of the $x$ and $y$ axis translations.

One odometer gives the number of turns made by one of the vehicle wheel. The mean value of the different odometers of the vehicle gives us the motion $(L)$ of the vehicle along a curvilinear axis.

From the knowledge of the vehicle motion $(L)$, and the yaw angle variation $(\Delta \psi)$, between two instants $t_{1}$ et $t_{2}$, we can estimate the $T_{x}$ and $T_{y}$ translations as shown in Fig. 5. When the yaw angle variation $\Delta \psi$ is not zero, we can consider that the vehicle is moving along an arc of circle with radius $R$ and center $O$.

This can be expressed with the following equations:

$$
\left\{\begin{array}{l}
T_{x}=R \sin (\Delta \psi) \\
T_{y}=R-R \cos (\Delta \psi) \quad \text { avec } \quad R=\frac{L}{\Delta \psi}
\end{array}\right.
$$

The yaw angle variation is: $\Delta \psi=\psi\left(t_{2}\right)-\psi\left(t_{1}\right)$. Since we are interested in the yaw angle variation, we calculate $\Delta \psi$ 


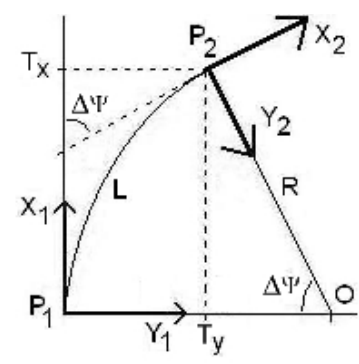

Fig. 5. Motion of the vehicle between two time $t_{1}\left(P_{1}\right)$ et $t_{2}\left(P_{2}\right)$

from $\dot{\psi}$ with a null initial condition $\left(\psi\left(t_{1}\right)=0\right)$, as far as we can can make the IMU frame superposed with $\left(P_{1}, x_{1}, y_{1}\right)$.

We are able to get, from the IMU and the odometers, an estimation of the $x$ and $y$-axis translations of the vehicle, if we know the pitch and roll angle. We can, if they are unknown, consider that they are small and then use the following approximation: $\dot{\psi}=r$.

Moreover the sensitivity analysis showed us that in some situations (indeed during braking and acceleration) the pitch angle is important.

\section{B. Real-Time Estimation of the Pitch and Roll Angle}

The vertical wheel forces approximations are calculated from longitudinal and transversal acceleration measures $\dot{v}_{x}$ and $\dot{v}_{y}$ of the vehicle by using the fundamental principle of dynamic [15]. The vehicle geometry is illustrated on the Fig. 6. The vertical wheel forces approximation $\hat{F}_{z i}$ are:

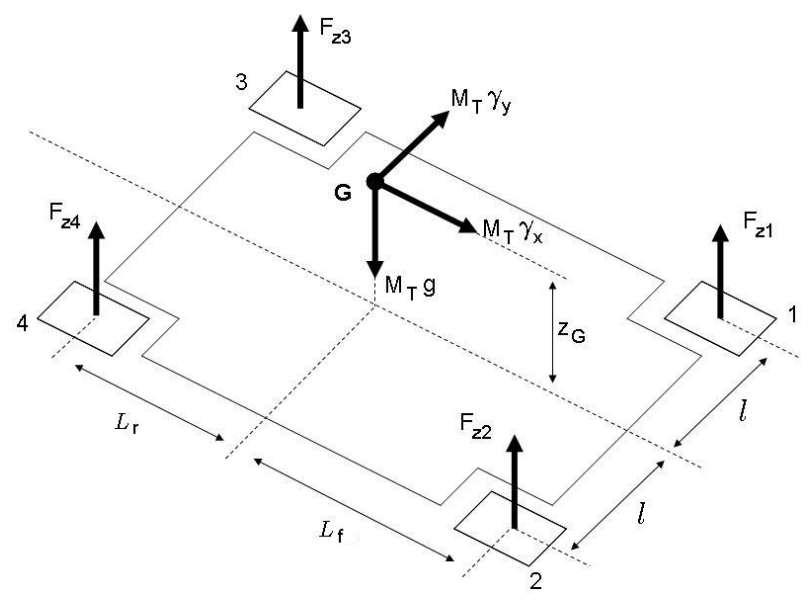

Fig. 6. Vehicle geometry

$$
\begin{aligned}
& \hat{F}_{z 1}=\frac{M_{T} L_{f}}{2\left(L_{f}+L_{r}\right)}\left(g+z_{g}\left(-\frac{\dot{v}_{y}}{l}-\frac{\dot{v}_{x}}{L_{f}}\right)\right) \\
& \hat{F}_{z 2}=\frac{M_{T} L_{f}}{2\left(L_{f}+L_{r}\right)}\left(g+z_{g}\left(\frac{\dot{v}_{y}}{l}-\frac{\dot{v}_{x}}{L_{f}}\right)\right) \\
& \hat{F}_{z 3}=\frac{M_{T} L_{r}}{2\left(L_{f}+L_{r}\right)} \\
& \hat{F}_{z 4}=\frac{M_{T} L_{r}}{2\left(L_{f}+L_{r}\right)}\left(g+z_{g}\left(-\frac{\dot{v}_{y}}{l}+\frac{\dot{v}_{x}}{L_{r}}\right)\right) \\
& \left.g+z_{g}\left(\frac{\dot{v}_{y}}{l}+\frac{\dot{v}_{x}}{L_{r}}\right)\right)
\end{aligned}
$$

where $M_{T}$ is the total weight of the vehicle, $L_{f}$ and $L_{r}$ are the distance from the center of gravity to the front and rear wheel, $g$ is the gravity acceleration, $2 l$ is the distance between the left and right wheel and $z_{g}$ is the height of the vehicle center of gravity.
This approximation allows us to estimate the wheel radius under load $\hat{z}_{r i}$ of each wheel, if the stiffness $k_{p}$ of the tyre is known and where $r_{l}$ is the wheel radius [15]:

$$
\hat{z}_{r i}=r_{l}-\frac{\hat{F}_{z i}}{k_{p}}
$$

The vehicle geometry is represented in Fig. 7. The values $z_{a i}$ are the wheel $i$ suspensions travel. As far as there is some vehicles equipped with suspension travel sensors, we will consider that we can measure those values. From

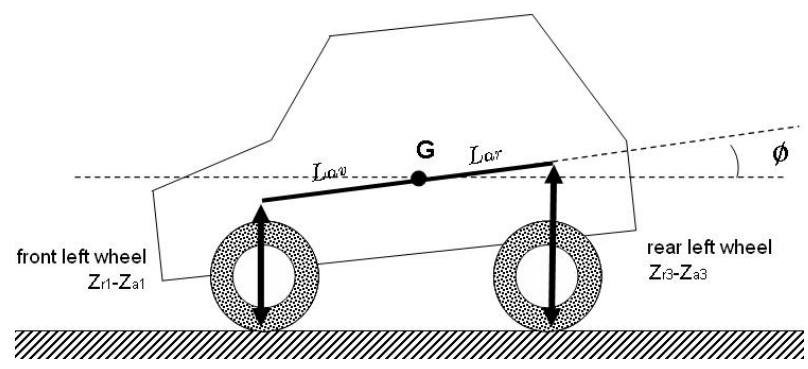

Fig. 7. Longitudinal geometry of the vehicle

the longitudinal geometry of the vehicle, we can obtain an estimation for left and right pitch:

$$
\begin{aligned}
& \hat{\phi}_{d}=\frac{\left(\hat{z}_{r 3}-z_{a 3}\right)-\left(\hat{z}_{r 1}-z_{a 1}\right)}{L_{a y}+L_{a r}} \\
& \hat{\phi}_{g}=\frac{\left(\hat{z}_{r 4}-z_{a 4}\right)-\left(\hat{z}_{r 2}-z_{a 2}\right)}{L_{a v}+L_{a r}}
\end{aligned}
$$

The vehicle pitch angle will be the mean of the left and right pitch angle. The same calculation can be done for the roll angle.

This method is interesting but needs to know the stiffness $k_{p}$ of the tyre and the total weight of the vehicle $M_{t}$. These constants are generally not well known.

We have tested this estimation methods with the simulator SiVIC, which takes into account the compression and the speed compression of tyres. We can see in Fig. 8, the result for the pitch angle estimation.

The difference between the reference and the estimation comes from the fact that the the tyre model used in the simulator is more complex than the one we use to do the estimation. This comes also from a simplifaction we have done. Indeed, we have simplified in (14) the vehicle acceleration $\dot{v}_{x}$ and $\dot{v}_{y}$ by the ones given by the IMU $a_{x}$ and $a_{y}$.

\section{RESULTS}

With the simulator SiVIC [16], we have simulated fog to reduce the visibility. We can see on the Fig. 9 results obtained with a meteorological visibility distance of $100 \mathrm{~m}$ (with fog) and without fog. We can see on Fig. 9 that for a $100 \mathrm{~m}$ visibility distance, our method gives result between 70 to 90 $\mathrm{m}$. When the visibility is good (no fog), we can see that we have an estimation of $300 \mathrm{~m}$ and more.

We have also realized some tests when the vehicle is braking. This will modify the pitch angle but we estimated it with (16). 


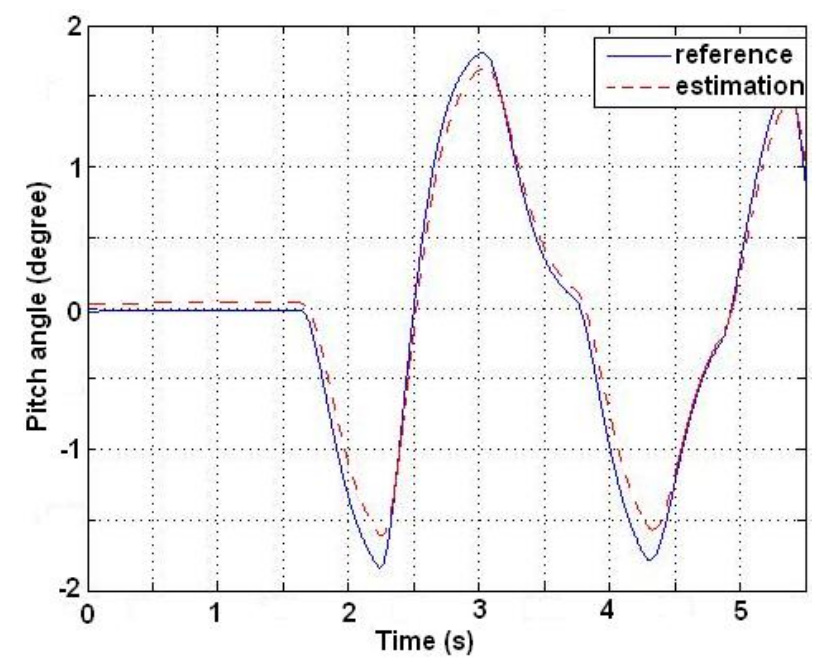

Fig. 8. Pitch angle estimation from the vertical dynamic of the vehicle.
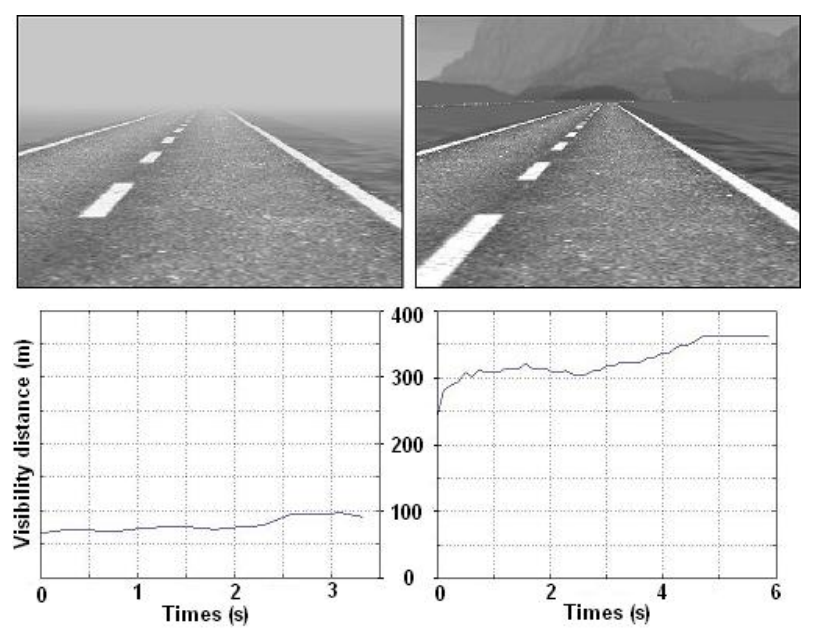

Fig. 9. Visibility distance results with the simulator SiVIC. On left, fog reducing the visibility distance to $100 \mathrm{~m}$. On right without fog.

We can see on Fig. 10 the result of the visibility distance estimation when the visibility is reduced to $50 \mathrm{~m}$. One can see that our estimation method gives result a bit shorter than $50 \mathrm{~m}$ when we estimate the pitch angle, in the contrary to the estimation made without the pitch angle estimation where we obtain false results.

\section{CONCLUSION}

We have presented here a study of the vehicle dynamics in the context of visibility distance estimation with successive image alignment.

A sensitivity analysis of the different degrees of freedom of the vehicle considered for the successive image alignment, allows us to determine relevant ones for our process and select the appropriate estimators.

Finally, some results and validation with a simulator were presented.

We see two prospectives to this work:

- We have presented in the introduction a method based on stereovision [3]. It would be interesting to compare

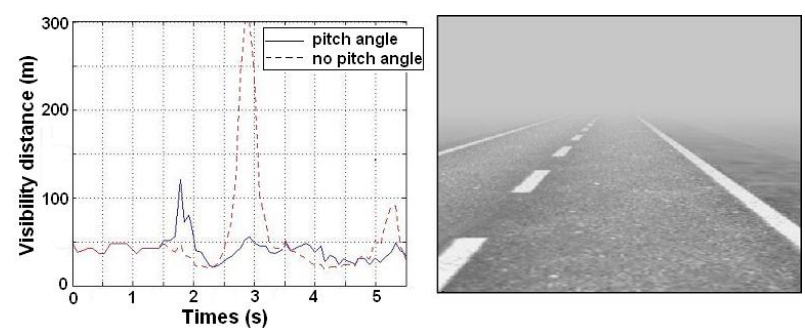

Fig. 10. Visibility distance estimation. In -.- with the pitch angle estimation and in - without.

the two methods.

- All the process presented here is based on flat world hypothesis at a $0 \mathrm{~m}$ height. An idea could be to consider several successive image alignment for several flat world hypothesis at different heights. If a point is not considered as part of the road, we could then see if it is not part of a different plane. We could then determine vertical elements and their heights.

\section{REFERENCES}

[1] D. Pomerleau, Visibility estimation from a moving vehicle using the RALPH vision system, IEEE Conference on Intelligent Transportation Systems, pp 906-911, November 1997.

[2] W. Middleton, Vision through the atmosphere, University of Toronto Press, 1952.

[3] N. Hautière and J.-P. Tarel and J. Lavenant and D. Aubert, Automatic Fog Detection and Estimation of Visibility Distance through use of an Onboard Camera, Machine Vision and Applications Journal, vol. 17, n. 1, pp 8-20, April 2006.

[4] R. Labayrade and D. Aubert, in-vehicle characterization of obstacles by stereovision, 1st International Workshop on In-Vehicle Cognitive Computer Vision Systems, Graz, Austria, 2003.

[5] N. Hautière and R. Labayrade and D. Aubert, Real-Time Disparity Contrast Combination for Onboard Estimation of the Visibility Distance, IEEE Transactions on Intelligent Transportation Systems, vol. 7, n. 2, June 2006.

[6] X. Brun and P. Charbonnier and F. Goulette, Modélisation 3D de routes par télémetrie laser embarquée pour la mesure de distance de visibilité, Journées des Sciences de l'Ingénieur, Marne-la-Vallée, France, December 2006.

[7] International lighting vocabulary, Commission Internationale de l'Eclairage, vol. 17.4, 1987.

[8] A. Shashua, Projective Structure from Uncalibrated Images: Structure from Motion and Recognition, IEEE Transactions on Pattern Analysis and Machine Intelligence, vol. 16, n. 8, pp 778-790, 2004.

[9] R. Horaud and O. Monga, "Vision par ordinateur, outils fondamentaux",Editions Hermès, 1995.

[10] M. Perrollaz, Construction d'une carte de disparité et application á la détection d'obstacles routiers, Technical Report, INRETS/LCPC, 2006.

[11] Leon M Arriola and J. M. Hyman, Forward and Adjoint Sensitivity Analysis : with Application in Dynamical System, Linear Algebra and Optimization, Technical report, Los Alamos National Laboratory, 2003.

[12] Todd A. Williamson, A High-Performance Stereo Vision System for Obstacle Detection, Carnegie Mellon University, september 1998.

[13] R. Khler, A segmentation system based on thresholding, Graphical Models and Image Processing, vol. 15, pp 319-338, 1981.

[14] C. Boussard and N. Hautière and D. Gruyer, Prototypage d'un capteur monoculaire générique de visibilité pour véhicule traceur, MajecSTIC 2006, Lorient, France, November 2006.

[15] H. Chou, Contribution au Contrôle Global de châssis, Ecole des Mines de Paris, December 2002.

[16] D. Gruyer and C. Royere and S. Glaser, SiVIC, une plate forme de prototypage d'environnement routier et de capteurs virtuels pour la conception et l'évaluation de systè mes d'aide la conduide, Journée Automatique Automobile, 8-9 November 2005. 